S. W. Kim, J. B. Lee and K. B. Lee

Nagoya Math. J.

Vol. 178 (2005), 37-53

\title{
AVERAGING FORMULA FOR NIELSEN NUMBERS
}

\author{
SEUNG WON KIM, JONG BUM LEE AND KYUNG BAI LEE
}

\begin{abstract}
We prove that the averaging formula for Nielsen numbers holds for continuous maps on infra-nilmanifolds: Let $M$ be an infra-nilmanifold and $f: M \rightarrow M$ be a continuous map. Suppose $M_{K}$ is a regular covering of $M$ which is a compact nilmanifold with $\pi_{1}\left(M_{K}\right)=K$. Assume that $f_{*}(K) \subset K$. Then $f$ has a lifting $\bar{f}: M_{K} \rightarrow M_{K}$ on $M_{K}$. We prove a question raised by McCord, which is for any $\alpha \in \pi_{1}(M)$ with $p(\operatorname{Fix}(\alpha \tilde{f}))$ an essential fixed point class, $\operatorname{fix}\left(\tau_{\alpha} \varphi\right)=1$. As a consequence, we obtain the following averaging formula for Nielsen numbers
\end{abstract}

$$
N(f)=\frac{1}{\left[\pi_{1}(M): K\right]} \sum_{\bar{\alpha} \in \pi_{1}(M) / K} N(\bar{\alpha} \bar{f}) .
$$

\section{$\S 1$. Introduction}

Let $X$ be a compact connected space, and let $\Pi$ be the group of covering transformations for the universal covering projection $p: \tilde{X} \rightarrow X$. For a normal subgroup $K$ of finite index in $\Pi$ we denote $\bar{X}=\tilde{X} / K, p^{\prime}: \tilde{X} \rightarrow \bar{X}$ and $\bar{p}: \bar{X} \rightarrow X$; then $p=\bar{p} \circ p^{\prime}$. Let $f: X \rightarrow X$ be a self-map. If $f_{*}(K) \subset K$, then $f$ has a lifting on $\bar{X}$. We will fix a lifting of $f$ on $\bar{X}$ and denote it by $\bar{f}: \bar{X} \rightarrow \bar{X}$. We will also fix a lifting of $f$ on $\tilde{X}$ and denote it by $\tilde{f}: \tilde{X} \rightarrow \tilde{X}$. Note that any lifting of $f$ on $\tilde{X}$ is of the form $\alpha \tilde{f}$ where $\alpha \in \Pi$, and any lifting of $f$ on $\bar{X}$ is of the form $\bar{\alpha} \bar{f}$ where $\bar{\alpha} \in \Pi / K$.

The Lefschetz number $L(f)$ of $f$ is defined by

$$
L(f)=\sum_{k}(-1)^{k} \operatorname{trace}\left\{\left(f_{*}\right)_{k}: H_{k}(X ; \mathbb{Q}) \rightarrow H_{k}(X ; \mathbb{Q})\right\}
$$

To define the Nielsen number $N(f)$ of $f$, we decompose the fixed point set $\operatorname{Fix}(f)=\{x \in X \mid f(x)=x\}$ of $f$ in a certain way into its subsets, called fixed point classes of $f$ (see Section 2). To each fixed point class $\mathbb{F}$, one

Received May 27, 2003.

2000 Mathematics Subject Classification: 55M20, 57S30.

Supported in part by the Sogang University Research Grant in 2003 and by grant No. R14-2002-044-01002-0(2003) from ABRL of KOSEF. 
can assign an integer $\operatorname{ind}(f, \mathbb{F})$. The fixed point class $\mathbb{F}$ is called essential if $\operatorname{ind}(f, \mathbb{F}) \neq 0$. The Nielsen number $N(f)$ of $f$ is defined to be

$$
N(f)=\text { the number of essential fixed point classes of } f \text {. }
$$

The Lefschetz number and Nielsen number are homotopy invariants. If $L(f) \neq 0$, then every map homotopic to $f$ has a fixed point. The Nielsen number $N(f)$ has the property that every map homotopic to $f$ has at least $N(f)$ fixed points. The Nielsen number gives more precise information concerning the existence of fixed points than the Lefschetz number, but its computation when compared with that of the Lefschetz number is in general much more difficult.

The following averaging formula for Lefschetz numbers is well-known [2, Theorem III.2.12]:

$$
L(f)=\frac{1}{[\Pi: K]} \sum_{\bar{\alpha} \in \Pi / K} L(\bar{\alpha} \bar{f}) .
$$

In this paper, we will deal with the averaging formula for Nielsen numbers. We prove in Theorem 3.1 that

$$
N(f) \geq \frac{1}{[\Pi: K]} \sum_{\bar{\alpha} \in \Pi / K} N(\bar{\alpha} \bar{f}) .
$$

We prove further that if $X$ is an infra-nilmanifold and $K$ consists of pure translations of $\Pi$, the above inequality becomes the equality. That is, we prove in Theorem 3.5 that the averaging formula for Nielsen numbers holds for continuous maps on infra-nilmanifolds.

\section{§2. Decomposition of the fixed point set}

For the fixed liftings $\bar{f}$ and $\tilde{f}$ of $f$, we have homomorphisms

$$
\begin{gathered}
\bar{\varphi}: \Pi / K \longrightarrow \Pi / K \quad \text { defined by } \bar{f} \bar{\alpha}=\bar{\varphi}(\bar{\alpha}) \bar{f}, \\
\varphi: \Pi \longrightarrow \Pi \quad \text { defined by } \tilde{f} \alpha=\varphi(\alpha) \tilde{f},
\end{gathered}
$$

so that $\varphi^{\prime}=\left.\varphi\right|_{K}: K \rightarrow K$ and the following diagram is commutative:

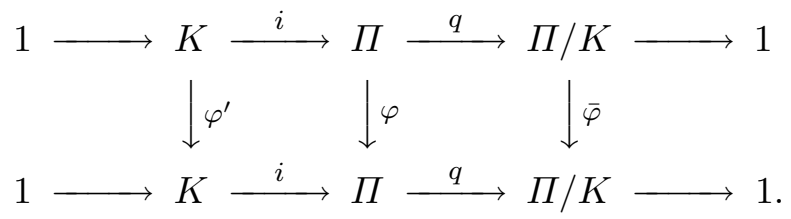


The homomorphism $\varphi: \Pi \rightarrow \Pi$ defines the Reidemeister action of $\Pi$ on $\Pi$ as follows:

$$
\Pi \times \Pi \longrightarrow \Pi, \quad(\gamma, \alpha) \longmapsto \gamma \alpha \varphi(\gamma)^{-1} .
$$

Similarly, the homomorphisms $\varphi^{\prime}: K \rightarrow K$ and $\bar{\varphi}: \Pi / K \rightarrow \Pi / K$ define the Reidemeister actions of $K$ on $K$ and $\Pi / K$ on $\Pi / K$, respectively. Denote the sets of Reidemeister classes of $K, \Pi, \Pi / K$ determined by $\varphi^{\prime}, \varphi, \bar{\varphi}$ by $\mathcal{R}\left[\varphi^{\prime}\right], \mathcal{R}[\varphi], \mathcal{R}[\bar{\varphi}]$, respectively.

The fixed point classes of $f$ are the subsets $p(\operatorname{Fix}(\alpha \tilde{f}))(\alpha \in \Pi)$ of the fixed point set $\operatorname{Fix}(f)$ of $f$. Each fixed point class $p(\operatorname{Fix}(\alpha \tilde{f}))$ is determined by the Reidemeister class $[\alpha] \in \mathcal{R}[\varphi]$, because $\alpha \tilde{f} \sim \gamma \alpha \varphi(\gamma)^{-1} \tilde{f}=\gamma \alpha \tilde{f} \gamma^{-1}$ for all $\gamma \in \Pi$ so that $p(\operatorname{Fix}(\alpha \tilde{f}))=p\left(\operatorname{Fix}\left(\gamma \alpha \tilde{f} \gamma^{-1}\right)\right)$. The fixed point set $\operatorname{Fix}(f)$ splits into a disjoint union of fixed point classes.

Now we have an exact sequence of sets

$$
\mathcal{R}\left[\varphi^{\prime}\right] \stackrel{\hat{i}}{\longrightarrow} \mathcal{R}[\varphi] \stackrel{\hat{q}}{\longrightarrow} \mathcal{R}[\bar{\varphi}] \longrightarrow 1,
$$

i.e., $\hat{q}$ is surjective and $\hat{q}^{-1}([\overline{1}])=\operatorname{im}(\hat{i})$. For each $\bar{\alpha} \in \Pi / K$ and $\alpha \in$ $q^{-1}(\bar{\alpha}), \alpha \tilde{f}$ is a lifting of $\bar{\alpha} \bar{f}$ and $f$, and $\bar{\alpha} \bar{f}$ is a lifting of $f$. They induce homomorphisms $\tau_{\alpha} \varphi, \tau_{\bar{\alpha}} \bar{\varphi}$ and $\tau_{\alpha} \varphi^{\prime}$, where $\tau_{\alpha}$ denotes the conjugation by $\alpha$, i.e.,

$$
\tau_{\alpha}(\beta)=\alpha \beta \alpha^{-1} \text {. }
$$

We denote the subgroup of $\Pi$ fixed by a homomorphism $\psi: \Pi \rightarrow \Pi$ by

$$
\operatorname{fix}(\psi)=\{\alpha \in \Pi \mid \psi(\alpha)=\alpha\} .
$$

Then the following diagram

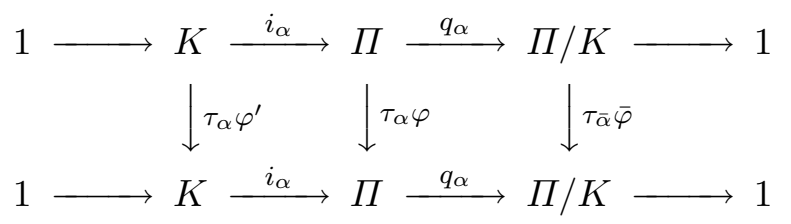

is commutative, and the following sequence of groups

$$
1 \longrightarrow \operatorname{fix}\left(\tau_{\alpha} \varphi^{\prime}\right) \stackrel{i_{\alpha}}{\longrightarrow} \operatorname{fix}\left(\tau_{\alpha} \varphi\right) \stackrel{q_{\alpha}}{\longrightarrow} \operatorname{fix}\left(\tau_{\bar{\alpha}} \bar{\varphi}\right)
$$

is exact, and the following sequence of sets

$$
\mathcal{R}\left[\tau_{\alpha} \varphi^{\prime}\right] \stackrel{\hat{i}_{\alpha}}{\longrightarrow} \mathcal{R}\left[\tau_{\alpha} \varphi\right] \stackrel{\hat{q}_{\alpha}}{\longrightarrow} \mathcal{R}\left[\tau_{\bar{\alpha}} \bar{\varphi}\right] \longrightarrow 1
$$

is exact. 
Lemma 2.1. For each $k \in K$ and $\alpha \in \Pi$, we have

(1) $\left|\hat{q}^{-1}([\bar{\alpha}])\right|=\left|\hat{q}_{\alpha}^{-1}([\overline{1}])\right|$,

(2) $|\mathcal{R}[\varphi]|=\sum_{[\bar{\alpha}] \in \mathcal{R}[\bar{\varphi}]}\left|\hat{q}^{-1}([\bar{\alpha}])\right|=\sum_{[\bar{\alpha}] \in \mathcal{R}[\bar{\varphi}]}\left|\hat{q}_{\alpha}^{-1}([\overline{1}])\right|=\sum_{[\bar{\alpha}] \in \mathcal{R}[\bar{\varphi}]}\left|\operatorname{im}\left(\hat{i}_{\alpha}\right)\right|$,

(3) $\left|\mathcal{R}\left[\tau_{\alpha} \varphi^{\prime}\right]\right|=\sum_{[k] \in \operatorname{im}\left(\hat{i}_{\alpha}\right)}\left|\hat{i}_{\alpha}^{-1}([k])\right|$,

(4) $\left|\hat{i}_{\alpha}^{-1}([k])\right|=\left[\operatorname{fix}\left(\tau_{\bar{\alpha}} \bar{\varphi}\right): q_{k \alpha}\left(\operatorname{fix}\left(\tau_{k \alpha} \varphi\right)\right)\right]$,

(5) $\left|\mathcal{R}\left[\tau_{\alpha} \varphi^{\prime}\right]\right|=\sum_{[k] \in \operatorname{im}\left(\hat{i}_{\alpha}\right)}\left[\operatorname{fix}\left(\tau_{\bar{\alpha}} \bar{\varphi}\right): q_{k \alpha}\left(\operatorname{fix}\left(\tau_{k \alpha} \varphi\right)\right)\right]$.

Proof. The first three equalities follow from the exactness of the following sets

$$
\begin{gathered}
\mathcal{R}\left[\varphi^{\prime}\right] \stackrel{\hat{i}}{\longrightarrow} \mathcal{R}[\varphi] \stackrel{\hat{q}}{\longrightarrow} \mathcal{R}[\bar{\varphi}] \longrightarrow 1, \\
\mathcal{R}\left[\tau_{\alpha} \varphi^{\prime}\right] \stackrel{\hat{i}_{\alpha}}{\longrightarrow} \mathcal{R}\left[\tau_{\alpha} \varphi\right] \stackrel{\hat{q}_{\alpha}}{\longrightarrow} \mathcal{R}\left[\tau_{\bar{\alpha}} \bar{\varphi}\right] \longrightarrow 1
\end{gathered}
$$

To prove (4), first we fix $\alpha \in \Pi$ and observe that

$$
\hat{i}_{\alpha}^{-1}([k])=\left\{\left[k^{\prime}\right] \in \mathcal{R}\left[\tau_{\alpha} \varphi^{\prime}\right]:\left[k^{\prime}\right]=[k] \in \mathcal{R}\left[\tau_{\alpha} \varphi\right]\right\} .
$$

Recall

$$
p(\operatorname{Fix}(k \tilde{f})) \longleftrightarrow[k] \in \mathcal{R}[\varphi]
$$

where $\varphi$ is induced by $\tilde{f}$. Since $\tau_{\alpha} \varphi$ corresponds to $\alpha \tilde{f}$, the above correspondence becomes

$$
p(\operatorname{Fix}(k \alpha \tilde{f})) \longleftrightarrow[k] \in \mathcal{R}\left[\tau_{\alpha} \varphi\right]
$$

Therefore

$$
\begin{aligned}
{\left[k^{\prime}\right] \in \hat{i}_{\alpha}^{-1}([k]) } & \Longleftrightarrow\left[k^{\prime}\right]=[k] \in \mathcal{R}\left[\tau_{\alpha} \varphi\right] \\
& \Longleftrightarrow p\left(\operatorname{Fix}\left(k^{\prime} \alpha \tilde{f}\right)\right)=p(\operatorname{Fix}(k \alpha \tilde{f})) .
\end{aligned}
$$

The commutative diagram

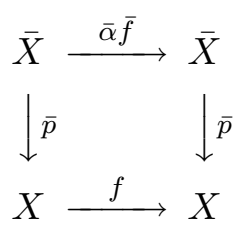


induces the following commutative diagrams

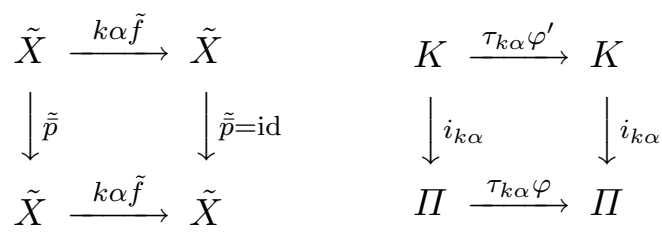

Then we have a well-defined map $\bar{p}_{\mathrm{FPC}}$

$$
\bar{p}_{\mathrm{FPC}}:\left\{p^{\prime}(\operatorname{Fix}(k \alpha \tilde{f})) \mid[k] \in \mathcal{R}\left[\tau_{\alpha} \varphi^{\prime}\right]\right\} \longrightarrow\left\{p(\operatorname{Fix}(k \alpha \tilde{f})) \mid[k] \in \mathcal{R}\left[\tau_{\alpha} \varphi\right]\right\}
$$

which sends a fixed point class $p^{\prime}(\operatorname{Fix}(k \alpha \tilde{f}))$ of $\bar{\alpha} \bar{f}$ to a fixed point class $p(\operatorname{Fix}(k \alpha \tilde{f}))$ of $f$. By the above observation, $\left|\hat{i}_{\alpha}^{-1}([k])\right|=\left|\bar{p}_{\mathrm{FPC}}^{-1}([k \alpha \tilde{f}])\right|$. Since $i_{k \alpha}: K \rightarrow \Pi$ is injective and $K$ is a normal subgroup of $\Pi$, we apply [2, Proposition III.1.15] to the above commutative diagrams and obtain

$$
\left|\hat{i}_{\alpha}^{-1}([k])\right|=\left|\bar{p}_{\text {FPC }}^{-1}([k \alpha \tilde{f}])\right|=\left[\operatorname{fix}\left(\tau_{\bar{\alpha}} \bar{\varphi}\right): q_{k \alpha}\left(\operatorname{fix}\left(\tau_{k \alpha} \varphi\right)\right)\right] .
$$

This proves (4), and (5) follows from (3) and (4).

The fixed point classes of $f$ are labelled by the Reidemeister classes $\mathcal{R}[\varphi]$ of $\varphi$. We may relabel them in terms of the Reidemeister classes $\mathcal{R}[\bar{\varphi}]$ and $\mathcal{R}\left[\tau_{\alpha} \varphi^{\prime}\right]$. This relabelling is useful in comparing the Nielsen number $N(f)$ of $f$ with the Nielsen numbers $N(\bar{\alpha} \bar{f})$ of $\bar{\alpha} \bar{f}$.

Lemma 2.2. (Decompositions of the fixed point sets) Let $f: X \rightarrow X$ be a self-map. Then

(1) $\bar{p}(\operatorname{Fix}(\bar{\alpha} \bar{f}))=\coprod_{[k] \in \operatorname{im}\left(\hat{i}_{\alpha}\right)} p(\operatorname{Fix}(k \alpha \tilde{f}))$,

(2) $\operatorname{Fix}(f)=\coprod_{[\bar{\alpha}] \in \mathcal{R}[\bar{\varphi}]} \coprod_{[k] \in \operatorname{im}\left(\hat{i}_{\alpha}\right)} p(\operatorname{Fix}(k \alpha \tilde{f}))$.

Proof. Recall first that the fixed point set of $f$ is the disjoint union of the $\bmod K$ fixed point classes of $f$, where the union ranges over the Reidemeister classes $[\bar{\alpha}] \in \mathcal{R}[\bar{\varphi}]$. I.e.,

$$
\operatorname{Fix}(f)=\coprod_{[\bar{\alpha}] \in \mathcal{R}[\bar{\varphi}]} \bar{p}(\operatorname{Fix}(\bar{\alpha} \bar{f}))
$$


For each $\alpha \in q^{-1}(\bar{\alpha})$,

$$
\operatorname{Fix}(\bar{\alpha} \bar{f})=\coprod_{[k] \in \mathcal{R}\left[\tau_{\alpha} \varphi^{\prime}\right]} p^{\prime}(\operatorname{Fix}(k \alpha \tilde{f}))
$$

Thus

$$
\begin{aligned}
\bar{p}(\operatorname{Fix}(\bar{\alpha} \bar{f})) & =\bigcup_{[k] \in \mathcal{R}\left[\tau_{\alpha} \varphi^{\prime}\right]} p(\operatorname{Fix}(k \alpha \tilde{f})), \\
\operatorname{Fix}(f) & =\coprod_{[\bar{\alpha}] \in \mathcal{R}[\bar{\varphi}]} \bigcup_{[k] \in \mathcal{R}\left[\tau_{\alpha} \varphi^{\prime}\right]} p(\operatorname{Fix}(k \alpha \tilde{f})) \\
& =\text { a union of fixed point classes of } f .
\end{aligned}
$$

In the proof of Lemma 2.1, $\left|\hat{i}_{\alpha}^{-1}([k])\right|=\left|\bar{p}_{\mathrm{FPC}}^{-1}([k \alpha \tilde{f}])\right|$. By Lemma 2.1.(3), the number of different fixed point classes $p(\operatorname{Fix}(k \alpha \tilde{f}))$ involved in the mod $K$ fixed point class $\bar{p}(\operatorname{Fix}(\bar{\alpha} \bar{f}))$ is $\left|\operatorname{im}\left(\hat{i}_{\alpha}\right)\right|$. Hence

$$
\begin{aligned}
\bar{p}(\operatorname{Fix}(\bar{\alpha} \bar{f})) & =\coprod_{[k] \in \operatorname{im}\left(\hat{i}_{\alpha}\right)} p(\operatorname{Fix}(k \alpha \tilde{f})), \\
\operatorname{Fix}(f) & =\coprod_{[\bar{\alpha}] \in \mathcal{R}[\bar{\varphi}]} \coprod_{[k] \in \operatorname{im}\left(\hat{i}_{\alpha}\right)} p(\operatorname{Fix}(k \alpha \tilde{f})) .
\end{aligned}
$$

This proves the result.

Remark 2.3. The group $\Pi / K$ acts on the set $[\bar{\alpha}] \in \mathcal{R}[\bar{\varphi}]$ by the rule $\bar{\alpha} \mapsto \bar{\beta} \bar{\alpha} \bar{\varphi}(\bar{\beta})^{-1}$. This action is transitive. The isotropy subgroup is

$$
\left\{\bar{\beta} \mid \bar{\beta} \bar{\alpha} \bar{\varphi}(\bar{\beta})^{-1}=\bar{\alpha}\right\}=\operatorname{fix}\left(\tau_{\bar{\alpha}} \bar{\varphi}\right) .
$$

Hence $[\Pi: K]=|[\bar{\alpha}]| \cdot\left|\operatorname{fix}\left(\tau_{\bar{\alpha}} \bar{\varphi}\right)\right|$.

Remark 2.4. For each $x \in \bar{p}(\operatorname{Fix}(\bar{\alpha} \bar{f}))$, we fix $\bar{x} \in \operatorname{Fix}(\bar{\alpha} \bar{f})$ with $\bar{p}(\bar{x})=$ $x$. Then $\operatorname{Fix}(\bar{\alpha} \bar{f}) \cap \bar{p}^{-1}(x)=\left\{\bar{\beta} \bar{x} \mid \bar{\beta} \in \operatorname{fix}\left(\tau_{\bar{\alpha}} \bar{\varphi}\right)\right\}$. That is, there are $\left|\operatorname{fix}\left(\tau_{\bar{\alpha}} \bar{\varphi}\right)\right|$ points in $\operatorname{Fix}(\bar{\alpha} \bar{f}) \cap \bar{p}^{-1}(x)$. Moreover,

$$
\begin{aligned}
L(\bar{\alpha} \bar{f}) & =\sum_{\bar{x} \in \operatorname{Fix}(\bar{\alpha} \bar{f})} \operatorname{ind}(\bar{\alpha} \bar{f}, \bar{x})=\left|\operatorname{fix}\left(\tau_{\bar{\alpha}} \bar{\varphi}\right)\right| \sum_{x \in \bar{p}(\operatorname{Fix}(\bar{\alpha} \bar{f}))} \operatorname{ind}(f, x) \\
& =\left|\operatorname{fix}\left(\tau_{\bar{\alpha}} \bar{\varphi}\right)\right| \cdot \operatorname{ind}(f, \bar{p}(\operatorname{Fix}(\bar{\alpha} \bar{f}))) .
\end{aligned}
$$


Lemma 2.5. Let $k \in K$ and $\alpha \in \Pi$. If $x \in p(\operatorname{Fix}(k \alpha \tilde{f}))$, then there are $\left|q_{k \alpha}\left(\operatorname{fix}\left(\tau_{k \alpha} \varphi\right)\right)\right|$ points in $p^{\prime}(\operatorname{Fix}(k \alpha \tilde{f})) \cap \bar{p}^{-1}(x)$. Hence,

$$
\left|p^{\prime}(\operatorname{Fix}(k \alpha \tilde{f}))\right|=\left|q_{k \alpha}\left(\operatorname{fix}\left(\tau_{k \alpha} \varphi\right)\right)\right| \cdot|p(\operatorname{Fix}(k \alpha \tilde{f}))| .
$$

Proof. Let $\bar{x} \in p^{\prime}(\operatorname{Fix}(\tilde{f}))$ and $\bar{p}(\bar{x})=x$. Then there exists $\tilde{x} \in \operatorname{Fix}(\tilde{f})$ such that $p(\tilde{x})=\bar{p}(\bar{x})=x$. If $\bar{\alpha} \bar{x} \in p^{\prime}(\operatorname{Fix}(\tilde{f}))$, then there exists $\tilde{x}^{\prime} \in \operatorname{Fix}(\tilde{f})$ such that $p\left(\tilde{x}^{\prime}\right)=\bar{p}(\bar{\alpha} \bar{x})=\bar{p}(\bar{x})=x$. Thus $\tilde{x}^{\prime}=\alpha \tilde{x}$ where $\alpha \in \operatorname{fix}(\varphi)$ and $q(\alpha)=\bar{\alpha}$. Thus for each $x \in p(\operatorname{Fix}(\tilde{f}))$ if we fix one $\bar{x} \in p^{\prime}(\operatorname{Fix}(\tilde{f}))$, then $p^{\prime}(\operatorname{Fix}(\tilde{f})) \cap \bar{p}^{-1}(x)=\{\bar{\alpha} \bar{x} \mid \bar{\alpha} \in q(\operatorname{fix}(\varphi))\}$. Thus for each $x \in p(\operatorname{Fix}(k \alpha \tilde{f}))$, there are $\left|q_{k \alpha}\left(\operatorname{fix}\left(\tau_{k \alpha} \varphi\right)\right)\right|$ points in $p^{\prime}(\operatorname{Fix}(k \alpha \tilde{f})) \cap \bar{p}^{-1}(x)$.

Definition 2.6. Let $k \in K$ and $\alpha \in \Pi$. Define $\epsilon_{k \alpha}^{\prime}$ and $\epsilon_{k \alpha}$ as follows:

$$
\begin{aligned}
& \epsilon_{k \alpha}^{\prime}= \begin{cases}0, & p^{\prime}(\operatorname{Fix}(k \alpha \tilde{f})) \text { is an inessential fixed point class of } \bar{\alpha} \bar{f} \\
1, & p^{\prime}(\operatorname{Fix}(k \alpha \tilde{f})) \text { is an essential fixed point class of } \bar{\alpha} \bar{f},\end{cases} \\
& \epsilon_{k \alpha}= \begin{cases}0, & p(\operatorname{Fix}(k \alpha \tilde{f})) \text { is an inessential fixed point class of } f ; \\
1, & p(\operatorname{Fix}(k \alpha \tilde{f})) \text { is an essential fixed point class of } f .\end{cases}
\end{aligned}
$$

Remark 2.7. The projections $p^{\prime}, p$ are local homeomorphisms, and the index is a local invariant. Thus by Lemma $2.5, p^{\prime}(\operatorname{Fix}(k \alpha \tilde{f}))$ is an essential fixed point class of $\bar{\alpha} \bar{f}$ if and only if $p(\operatorname{Fix}(k \alpha \tilde{f}))$ is an essential fixed point class of $f$. Thus $\epsilon_{k \alpha}^{\prime}=\epsilon_{k \alpha}$.

In all, we have the following natural one-to-one correspondences:

$$
\begin{aligned}
& \coprod_{\bar{\alpha} \in \Pi / K} \coprod_{[k] \in \mathcal{R}\left[\tau_{\alpha} \varphi^{\prime}\right]} p^{\prime}(\operatorname{Fix}(k \alpha \tilde{f})) \\
\longleftrightarrow & \coprod_{\bar{\alpha} \in \Pi / K} \coprod_{[k] \in \mathcal{R}\left[\tau_{\alpha} \varphi^{\prime}\right]}\left|q_{k \alpha}\left(\operatorname{fix}\left(\tau_{k \alpha} \varphi\right)\right)\right| \cdot p(\operatorname{Fix}(k \alpha \tilde{f})) \\
\longleftrightarrow & {[\Pi: K] \coprod_{\bar{\alpha} \in \Pi / K} \coprod_{[k] \in \mathcal{R}\left[\tau_{\alpha} \varphi^{\prime}\right]} \frac{\left|q_{k \alpha}\left(\operatorname{fix}\left(\tau_{k \alpha} \varphi\right)\right)\right|}{|[\bar{\alpha}]| \cdot\left|\operatorname{fix}\left(\tau_{\bar{\alpha}} \bar{\varphi}\right)\right|} p(\operatorname{Fix}(k \alpha \tilde{f})) }
\end{aligned}
$$

(by Remark 2.3)

$$
\begin{aligned}
& \longleftrightarrow \quad[\Pi: K] \coprod_{\bar{\alpha} \in \Pi / K} \frac{1}{|[\bar{\alpha}]|} \coprod_{[k] \in \mathcal{R}\left[\tau_{\alpha} \varphi^{\prime}\right]} \frac{p(\operatorname{Fix}(k \alpha \tilde{f}))}{\left[\operatorname{fix}\left(\tau_{\bar{\alpha}} \bar{\varphi}\right): q_{k \alpha}\left(\operatorname{fix}\left(\tau_{k \alpha} \varphi\right)\right)\right]} \\
& \longleftrightarrow[\Pi: K] \coprod_{[\bar{\alpha}] \in \mathcal{R}[\bar{\varphi}]} \coprod_{[k] \in \operatorname{im}\left(\hat{i}_{\alpha}\right)} p(\operatorname{Fix}(k \alpha \tilde{f})) \quad \text { by Lemma 2.1. }
\end{aligned}
$$




\section{§3. Averaging formula for Nielsen numbers}

Let $g: X \rightarrow X$ be another self-map such that $g_{*}(K) \subset K$. Let $\bar{g}: \bar{X} \rightarrow$ $\bar{X}$ be a fixed lifting of $g, \tilde{g}: \tilde{X} \rightarrow \tilde{X}$ a fixed lifting of $\bar{g}$, and $\psi: \Pi \rightarrow \Pi$ the homomorphism induced by $\tilde{g}$. The coincidence set Coin $(f, g)=\{x \in$ $X \mid f(x)=g(x)\}$ of $f$ and $g$ can be decomposed into its subsets, called coincidence classes, in a similar manner as in the fixed point case. When $X$ is a closed orientable manifold, one can assign to each coincidence class the index. A coincidence class is essential if its index is nonzero. The Nielsen coincidence number $N(f, g)$ is, by definition, the number of essential coincidence classes.

C. McCord proves in [5, Corollary 5.10] that if, for any $k \in K$ and $\alpha \in \Pi$ with $p(\operatorname{Coin}(k \alpha \tilde{f}, \tilde{g}))$ an essential coincidence class, the subgroup $\operatorname{coin}\left(\tau_{k \alpha} \varphi, \psi\right)=\left\{\beta \in \Pi \mid \tau_{k \alpha} \varphi(\beta)=\psi(\beta)\right\}$ of $\Pi$ is contained in $K$, then

$$
N(f, g)=\frac{1}{[\Pi: K]} \sum_{\bar{\alpha} \in \Pi / K} N(\bar{\alpha} \bar{f}, \bar{g}) .
$$

He also raises a question in [5] whether for any $k \in K$ and $\alpha \in \Pi$ with $p(\operatorname{Coin}(k \alpha \tilde{f}, \tilde{g}))$ an essential coincidence class, $\operatorname{coin}\left(\tau_{k \alpha} \varphi, \psi\right)=1$ on all infra-nilmanifolds.

In Theorem 3.1 below, we prove that the fixed point version of the above is not only necessary but also sufficient. Furthermore, in Theorem 3.5 we prove that McCord's question for fixed point version is true and as a consequence, we obtain an averaging formula for Nielsen fixed point numbers on all infra-nilmanifolds.

THEOREM 3.1. Let $f: X \rightarrow X$ be a self-map on a compact connected space $X$, and let $K$ be a normal subgroup of $\Pi=\pi_{1}(X)$ of finite index such that $f_{*}(K) \subset K$. Then

$$
N(f) \geq \frac{1}{[\Pi: K]} \sum_{\bar{\alpha} \in \Pi / K} N(\bar{\alpha} \bar{f})
$$

and equality occurs if and only if for each $k \in K$ and $\alpha \in \Pi$ with $p(\operatorname{Fix}(k \alpha \tilde{f}))$ an essential fixed point class, $\left|q_{k \alpha}\left(\operatorname{fix}\left(\tau_{k \alpha} \varphi\right)\right)\right|=1$.

Proof. Our proof uses the one-to-one correspondences of Remark 2.7. 
Then

$$
\begin{aligned}
N(f) & =\sum_{[\bar{\alpha}] \in \mathcal{R}[\bar{\varphi}]} \sum_{[k] \in \operatorname{im}\left(\hat{i}_{\alpha}\right)} \epsilon_{k \alpha} \\
& =\sum_{\bar{\alpha} \in \Pi / K} \frac{1}{|[\bar{\alpha}]|} \sum_{[k] \in \mathcal{R}\left[\tau_{\alpha} \varphi^{\prime}\right]} \frac{1}{\left[\operatorname{fix}\left(\tau_{\bar{\alpha}} \bar{\varphi}\right): q_{k \alpha}\left(\operatorname{fix}\left(\tau_{k \alpha} \varphi\right)\right)\right]} \epsilon_{k \alpha} \\
& =\sum_{\bar{\alpha} \in \Pi / K} \sum_{[k] \in \mathcal{R}\left[\tau_{\alpha} \varphi^{\prime}\right]} \frac{\left|q_{k \alpha}\left(\operatorname{fix}\left(\tau_{k \alpha} \varphi\right)\right)\right|}{|[\bar{\alpha}]| \cdot\left|\operatorname{fix}\left(\tau_{\bar{\alpha}} \bar{\varphi}\right)\right|} \epsilon_{k \alpha} \\
& =\frac{1}{[\Pi: K]} \sum_{\bar{\alpha} \in \Pi / K} \sum_{[k] \in \mathcal{R}\left[\tau_{\alpha} \varphi^{\prime}\right]}\left|q_{k \alpha}\left(\operatorname{fix}\left(\tau_{k \alpha} \varphi\right)\right)\right| \epsilon_{k \alpha} \\
& =\frac{1}{[\Pi: K]} \sum_{\bar{\alpha} \in \Pi / K} \sum_{[k] \in \mathcal{R}\left[\tau_{\alpha} \varphi^{\prime}\right]}\left|q_{k \alpha}\left(\operatorname{fix}\left(\tau_{k \alpha} \varphi\right)\right)\right| \epsilon_{k \alpha}^{\prime} \\
& \geq \frac{1}{[\Pi: K]} \sum_{\bar{\alpha} \in \Pi / K[k] \in \mathcal{R}\left[\tau_{\alpha} \varphi^{\prime}\right]} \epsilon_{k \alpha} \\
& =\frac{1}{[\Pi: K]} \sum_{\bar{\alpha} \in \Pi / K} \sum_{N \bar{\alpha}) .}
\end{aligned}
$$

Moreover, the equality holds if and only if for each $k \in K$ and $\alpha \in \Pi$, either $\epsilon_{k \alpha}=0$ or $\left|q_{k \alpha}\left(\operatorname{fix}\left(\tau_{k \alpha} \varphi\right)\right)\right|=1$.

Corollary 3.2. Let $f: X \rightarrow X$ be a self-map on a compact connected space $X$ with finite fundamental group $\Pi$, then

$$
N(f)=\frac{1}{|\Pi|} \sum_{\alpha \in \Pi}\left|\operatorname{fix}\left(\tau_{\alpha} \varphi\right)\right| \cdot N(\alpha \tilde{f}) \geq \frac{1}{|\Pi|} \sum_{\alpha \in \Pi} N(\alpha \tilde{f})
$$

and equality occurs if and only if for each $\alpha \in \Pi$ with $p(\operatorname{Fix}(\alpha \tilde{f}))$ an essential fixed point class, $\left|\operatorname{fix}\left(\tau_{\alpha} \varphi\right)\right|=1$.

ExAmple 3.3. Let $f: \mathbb{R} P^{2} \rightarrow \mathbb{R} P^{2}$ be the identity map and $\tilde{f}: S^{2} \rightarrow$ $S^{2}$ be the identity map, i.e., $f=\mathrm{id}_{\mathbb{R} P^{2}}$ and $\tilde{f}=\mathrm{id}_{S^{2}}$. Then we have a commutative diagram

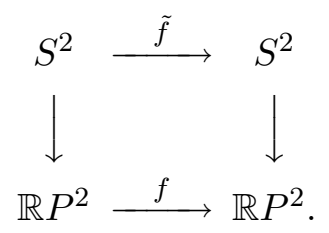


The group of deck transformations is $\Pi=\{1, \alpha\} \cong \mathbb{Z}_{2}$. The homomorphism $\varphi: \Pi \rightarrow \Pi$ corresponding to $\tilde{f}$ is the identity map. In particular, $\operatorname{fix}(\varphi)=$ $\Pi$.

Note that $\alpha \tilde{f}=\alpha$ is the antipodal map on $S^{2}$. This map has no fixed point; $\operatorname{Fix}(\alpha \tilde{f})=\emptyset ; p(\operatorname{Fix}(\alpha \tilde{f}))$ is an inessential fixed point class of $f$ and $N(\alpha \tilde{f})=0$.

Next we compute $N(\tilde{f})$ and find out whether or not $p(\operatorname{Fix}(\tilde{f}))$ is an essential fixed point class of $\operatorname{Fix}(f)$. By Remark 2.4 with $K=\{1\}$, we have $L(\tilde{f})=|\operatorname{fix}(\varphi)| \cdot \operatorname{ind}(f, p(\operatorname{Fix}(\tilde{f})))$. Thus

$$
2=L(\tilde{f})=|\operatorname{fix}(\varphi)| \cdot \operatorname{ind}(f, p(\operatorname{Fix}(\tilde{f})))=2 \cdot \operatorname{ind}(f, p(\operatorname{Fix}(\tilde{f}))) .
$$

Hence $p(\operatorname{Fix}(\tilde{f}))$ is an essential fixed point class of $f$ and $N(\tilde{f})=1$.

In all, $p(\operatorname{Fix}(\tilde{f}))$ is an essential fixed point class and $p(\operatorname{Fix}(\alpha \tilde{f}))$ is an inessential fixed point class of $f$. Hence $N(f)=1$, but $\frac{1}{|\Pi|} \sum_{\alpha \in \Pi} N(\alpha \tilde{f})=$ $\frac{1}{2}(N(\tilde{f})+N(\alpha \tilde{f}))=\frac{1}{2}(1+0)=\frac{1}{2}$. This shows that $N(f) \neq \frac{1}{|\Pi|} \sum_{\alpha \in \Pi} N(\alpha \tilde{f})$.

Now we prove the averaging formula for Nielsen numbers for continuous maps on infra-nilmanifolds. First we recall the following.

Lemma 3.4. ([4, Theorem 1.1]) Let $\Pi, \Pi^{\prime} \subset G \rtimes \operatorname{Aut}(G)$ be two almost crystallographic groups. Then for any homomorphism $\theta: \Pi \rightarrow \Pi^{\prime}$, there exists $(d, D) \in G \rtimes \operatorname{Endo}(G)$ such that for all $\alpha \in \Pi$,

$$
\theta(\alpha) \cdot(d, D)=(d, D) \cdot \alpha
$$

THEOREM 3.5. Let $M$ be an infra-nilmanifold and $f: M \rightarrow M$ be any self map. Suppose $M_{K}$ is a regular covering of $M$ which is a compact nilmanifold with $\pi_{1}\left(M_{K}\right)=K$. Assume that $f_{*}(K) \subset K$. Then

$$
N(f)=\frac{1}{[\Pi: K]} \sum N(\bar{f}),
$$

where the sum ranges over all the liftings $\bar{f}$ of $f$ onto $M_{K}$. In particular, $N(f) \geq|L(f)|$.

Proof. Let $M=\Pi \backslash G$ be the infra-nilmanifold. Then $K \subset \Pi \cap G$ and $M_{K}=K \backslash G$. Now we fix a lifting $\tilde{f}: G \rightarrow G$ of $f: M \rightarrow M$. Then $\tilde{f}$ induces a homomorphism $\varphi: \Pi \rightarrow \Pi$ on the group $\Pi$ of covering transformations of $p: G \rightarrow M$. Namely, for any $\alpha \in \Pi, \varphi(\alpha) \tilde{f}=\tilde{f} \alpha$. Since $\varphi: \Pi \rightarrow \Pi$ 
induces $\varphi^{\prime}: K \rightarrow K$, there is a continuous map $\bar{f}: M_{K} \rightarrow M_{K}$ which makes the following diagram

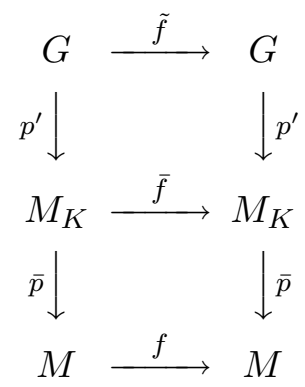

commutative.

By Lemma 3.4, there exists $(d, D) \in G \rtimes \operatorname{Endo}(G)$ such that

$$
\varphi(\alpha) \cdot(d, D)=(d, D) \cdot \alpha
$$

for all $\alpha \in \Pi$. This implies that the map $(d, D): G \rightarrow G$ induces a map $\Phi_{(d, D)}: M \rightarrow M$, and furthermore, $\Phi_{(d, D)}$ and $f$ induce exactly the same homomorphism $\varphi$ on $\Pi$. Since $M$ is a $K(\Pi, 1)$-manifold, $\Phi_{(d, D)}$ and $f$ are homotopic. By the homotopy invariance of the Nielsen number, we can replace $\tilde{f}, \bar{f}$ and $f$ in the diagram by $(d, D), \phi_{(d, D)}$ and $\Phi_{(d, D)}$, respectively, in the following discussion.

Write $E=\tau_{d} D \in \operatorname{Aut}(G)$, where $\tau_{d}$ is conjugation by $d$. The above equality yields

$$
\varphi^{\prime}(k)=\varphi(k)=\left(\tau_{d} D\right)(k) \text { for all } k \in K \text {. }
$$

Thus the map $E: G \rightarrow G$ yields a map $\phi_{E}: M_{K} \rightarrow M_{K}$ on the nilmanifold $M_{K}$, and $\bar{f} \simeq \phi_{E}$. By [1], $N(\bar{f})=\left|\operatorname{det}\left(I-E_{*}\right)\right|$, where $E_{*}$ is the linear map on the Lie algebra of the Lie group $G$ induced by $E$. Since $\operatorname{det}\left(I-E_{*}\right)=$ $\operatorname{det}\left(I-\operatorname{Ad}(d) D_{*}\right)=\operatorname{det}\left(I-D_{*}\right)\left(\left[3\right.\right.$, Lemma 1.2]), $N(\bar{f})=\left|\operatorname{det}\left(I-D_{*}\right)\right|$, and hence $N(\bar{f}) \neq 0$ if and only if $\operatorname{fix}\left(D_{*}\right)=\{0\}$ if and only if $\operatorname{Fix}(D)=\{e\}$.

Now we observe the following: Let $F: G \rightarrow G$ be any homomorphism on $G$ and $g \in G$. If $\operatorname{Fix}((g, F)) \neq \emptyset$, then there is a one-to-one correspondence between $\operatorname{Fix}((g, F))$ and $\operatorname{Fix}(F)$. Let $h \in \operatorname{Fix}((g, F))$. Then $(h, I)^{-1}(g, F)(h, I)=(e, F)=F$ and thus $k \in \operatorname{Fix}((g, F)) \mapsto h^{-1} k \in$ $\operatorname{Fix}(F)$ is the required correspondence. Therefore, $\operatorname{Fix}((g, F))=h \cdot \operatorname{Fix}(F)$.

Since $k \alpha \tilde{f}=(k, I)(a, A)(d, D)=(k \cdot a \cdot A(d), A D)$ where $\alpha=(a, A)$, $\bar{\alpha} \bar{f}=\phi_{(k \cdot a \cdot A(d), A D)}$ and $N(\bar{\alpha} \bar{f})=\left|\operatorname{det}\left(I-A_{*} D_{*}\right)\right|$. Therefore if $N(\bar{\alpha} \bar{f}) \neq 0$, the above observation implies that for all $k \in K, \operatorname{Fix}(k \alpha \tilde{f})$ has only one 
point. It follows that $\operatorname{fix}\left(\tau_{k \alpha} \varphi\right)=\{1\}$ and so $\left|q_{k \alpha}\left(\operatorname{fix}\left(\tau_{k \alpha} \varphi\right)\right)\right|=1$ for all $k \in K$. On the other hand, if $N(\bar{\alpha} \bar{f})=0$, then $\epsilon_{k \alpha}=0$ so that the fixed point class $p(\operatorname{Fix}(k \alpha \tilde{f}))$ of $f$ is inessential. Hence our result follows from Theorem 3.1.

Remark 3.6. In Theorem 3.5, we are assuming that $f: M \rightarrow M$ and $K$ is a finite-index, normal subgroup of $\Pi$ consisting of pure translations of the infra-nilmanifold $M$ so that $f_{*}(K) \subset K$. However it is known that such a $K$ always exists. In fact, it is shown in [3, Lemma 1.1] that there exists a fully invariant subgroup $K \subset \Gamma$ of $\pi_{1}(M)$ which is of finite index. Therefore for any $f: M \rightarrow M, f_{*}(K) \subset K$.

Remark 3.7. Let $f: M \rightarrow M$ be a continuous map on the infranilmanifold $M$. Let $K$ be a normal subgroup of $\pi_{1}(M)$ of finite index consisting of pure translations such that $f_{*}(K) \subset K$.

As we have seen in the proof of Theorem 3.5, we may assume that $f$ is the map $\Phi_{(d, D)}$ induced by an affine map $(d, D): G \rightarrow G$. Suppose $N(\bar{\alpha} \bar{f}) \neq 0$. Then for all $k \in K, \operatorname{Fix}(k \alpha \tilde{f})$ has only one point. Hence all the fixed point classes $p^{\prime}(\operatorname{Fix}(k \alpha \tilde{f}))$ of $\bar{\alpha} \bar{f}: M_{K} \rightarrow M_{K}$ on the nilmanifold $M_{K}$ have only one point and they all have the same index $\pm 1=\frac{L(\bar{\alpha} \bar{f})}{N(\bar{\alpha} f)}$. Therefore, if some $\epsilon_{k \alpha}^{\prime}=1$ then $\epsilon_{k \alpha}^{\prime}=1$ for all $k \in K$.

By Remark 2.7, $\epsilon_{k \alpha}^{\prime}=\epsilon_{k \alpha}$. Hence if some $\epsilon_{k \alpha}=1$, then $\epsilon_{k \alpha}=1$ for all $k \in K$.

It is clear from the definition of mod $K$ fixed point class that if every essential mod $K$ fixed point class coincides with an ordinary fixed point class, then $N_{K}(f)=N(f)$. We show that the converse is also true for every continuous maps on infra-nilmanifolds.

Corollary 3.8. Let $f: M \rightarrow M$ be any self-map on an infra-nilmanifold $M$. Let $M_{K}$ be a regular covering of $M$ which is a compact nilmanifold with $\pi_{1}\left(M_{K}\right)=K$. Assume $f_{*}(K) \subset K$. Then

$$
N(f) \geq N_{K}(f),
$$

and equality occurs if and only if every essential mod $K$ fixed point class coincides with an ordinary fixed point class of $f$. 
Proof. By Lemma 2.2.(1), $\bar{p}(\operatorname{Fix}(\bar{\alpha} \bar{f}))=\coprod_{[k] \in \operatorname{im}\left(\hat{i}_{\alpha}\right)} p(\operatorname{Fix}(k \alpha \tilde{f}))$. That is, the mod $K$ fixed point class $\bar{p}(\operatorname{Fix}(\bar{\alpha} \bar{f}))$ contains exactly $\left|\operatorname{im}\left(\hat{i}_{\alpha}\right)\right|$ ordinary fixed point classes $p(\operatorname{Fix}(k \alpha \tilde{f}))$ of $f$. Define $\epsilon_{\bar{\alpha}}$ as follows:

$$
\epsilon_{\bar{\alpha}}= \begin{cases}0, & \text { the } \bmod K \text { fixed point class } \bar{p}(\operatorname{Fix}(\bar{\alpha} \bar{f})) \text { of } f \text { is inessential; } \\ 1, & \text { the } \bmod K \text { fixed point class } \bar{p}(\operatorname{Fix}(\bar{\alpha} \bar{f})) \text { of } f \text { is essential. }\end{cases}
$$

By Remark 3.7, $\epsilon_{\bar{\alpha}}=1$ if and only if all $\epsilon_{k \alpha}^{\prime}=1$. Then $\epsilon_{\bar{\alpha}} \leq\left|\operatorname{im}\left(\hat{i}_{\alpha}\right)\right| \cdot \epsilon_{k \alpha}^{\prime}$. Hence

$$
\begin{aligned}
N(f) & =\sum_{[\bar{\alpha}] \in \mathcal{R}[\bar{\varphi}]} \sum_{[k] \in \operatorname{im}\left(\hat{i}_{\alpha}\right)} \epsilon_{k \alpha}=\sum_{[\bar{\alpha}] \in \mathcal{R}[\bar{\varphi}]} \sum_{[k] \in \operatorname{im}\left(\hat{i}_{\alpha}\right)} \epsilon_{k \alpha}^{\prime} \\
& =\sum_{[\bar{\alpha}] \in \mathcal{R}[\bar{\varphi}]}\left|\operatorname{im}\left(\hat{i}_{\alpha}\right)\right| \cdot \epsilon_{k \alpha}^{\prime} \geq \sum_{[\bar{\alpha}] \in \mathcal{R}[\bar{\varphi}]} \epsilon_{\bar{\alpha}}=N_{K}(f) .
\end{aligned}
$$

Thus $N_{K}(f)=N(f)$ if and only if for $\epsilon_{\bar{\alpha}}=1,\left|\operatorname{im}\left(\hat{i}_{\alpha}\right)\right|=1$ if and only if every essential mod $K$ fixed point class coincides with an ordinary fixed point class of $f$.

The next proposition tells us how to compute $\left|\operatorname{im}\left(\hat{i}_{\alpha}\right)\right|$.

Proposition 3.9. Let $f: M \rightarrow M$ be any self-map on an infranilmanifold $M$. Suppose $M_{K}$ is a regular covering of $M$ which is a compact nilmanifold with $\pi\left(M_{K}\right)=K$. Assume that $f_{*}(K) \subset K$. Then every essential mod $K$ fixed point class $\bar{p}(\operatorname{Fix}(\bar{\alpha} \bar{f}))$ contains exactly $\left|\operatorname{im}\left(\hat{i}_{\alpha}\right)\right|$ ordinary fixed point classes of $f$, where

$$
\left|\operatorname{im}\left(\hat{i}_{\alpha}\right)\right|=\frac{N(\bar{\alpha} \bar{f})}{\left|\operatorname{fix}\left(\tau_{\bar{\alpha}} \bar{\varphi}\right)\right|}=\frac{N(\bar{\alpha} \bar{f})}{[\Pi: K]} \cdot|[\bar{\alpha}]| .
$$

Proof. By Lemma 2.1.(4) and by the last paragraph of the proof of Theorem 3.5,

$$
\left|\hat{i}_{\alpha}^{-1}([k])\right|=\left[\operatorname{fix}\left(\tau_{\bar{\alpha}} \bar{\varphi}\right): q_{k \alpha}\left(\operatorname{fix}\left(\tau_{k \alpha} \varphi\right)\right)\right]=\left|\operatorname{fix}\left(\tau_{\bar{\alpha}} \bar{\varphi}\right)\right|,
$$

which is independent of $k$. Since, by Lemma 2.1.(3),

$$
\left|\mathcal{R}\left[\tau_{\alpha} \varphi^{\prime}\right]\right|=\sum_{[k] \in \operatorname{im}\left(\hat{i}_{\alpha}\right)}\left|\hat{i}_{\alpha}^{-1}([k])\right|
$$


and since, by Remark 2.3, $[\Pi: K]=|[\bar{\alpha}]| \cdot \mid$ fix $\left(\tau_{\bar{\alpha}} \bar{\varphi}\right) \mid$, we have

$$
\begin{aligned}
\left|\operatorname{im}\left(\hat{i}_{\alpha}\right)\right| & =\frac{\left|\mathcal{R}\left[\tau_{\alpha} \varphi^{\prime}\right]\right|}{\left|\hat{i}_{\alpha}^{-1}([k])\right|}=\frac{\left|\mathcal{R}\left[\tau_{\alpha} \varphi^{\prime}\right]\right|}{\mid \operatorname{fix}\left(\tau_{\bar{\alpha} \bar{\varphi}) \mid}\right.} \\
& =\frac{N(\bar{\alpha} \bar{f})}{\left|\operatorname{fix}\left(\tau_{\bar{\alpha}} \bar{\varphi}\right)\right|}=\frac{N(\bar{\alpha} \bar{f})}{[\Pi: K]} \cdot|[\bar{\alpha}]| .
\end{aligned}
$$

This completes our result.

Example 3.10. Let $G$ be the 3-dimensional Heisenberg group. That is,

$$
G=\left\{\left[\begin{array}{lll}
1 & x & z \\
0 & 1 & y \\
0 & 0 & 1
\end{array}\right]: x, y, z \in \mathbb{R}\right\}
$$

We denote this general element by $\{x, y, z\}$. Let $\Gamma$ be the subgroup of $G$ which is generated by

$$
\left\{0,0, \frac{1}{2}\right\}, \quad\{0,1,0\}, \quad\{1,0,0\} \text {. }
$$

Then $\Gamma$ is a subgroup of $G$ and in fact it is a lattice of $G$. by

Let $a=\left\{0,0, \frac{1}{2}\right\} \in G$ and $A: G \rightarrow G$ be the automorphism of $G$ given

$$
A(\{x, y, z\})=\{-x,-y, z\} .
$$

Then $A$ has period 2, and $(a, A)^{2}=\left(a^{2}, I\right)=(\{0,0,1\}, I) \in G \rtimes \operatorname{Aut}(G)$, where $I$ is the identity automorphism of $G$. The subgroup

$$
\Pi=\langle\Gamma,(a, A)\rangle \subset G \rtimes \operatorname{Aut}(G)
$$

generated by the lattice $\Gamma$ and the element $(a, A)$ is discrete and torsion free, and $\Gamma$ is a normal subgroup of $\Pi$ of index 2 . Thus $\Pi$ is an almost Bieberbach group, and $\Pi \backslash G$ is an infra-nilmanifold, which has a double covering $\Gamma \backslash G \rightarrow \Pi \backslash G$ by its holonomy group, $\Psi=\Pi / \Gamma=\{1, A\} \cong \mathbb{Z}_{2}$.

Let $D: G \rightarrow G$ be the automorphism of $G$ given by (cf. [6, pp.276-277])

$$
D(\{x, y, z\})=\left\{x+y, x,-z+\frac{1}{2} x^{2}+x y\right\} .
$$

Then $D A=A D$ and the conjugation by $(\{0,0,0\}, D) \in G \rtimes \operatorname{Aut}(G)$ maps $\Pi$ into $\Pi$ (and $\Gamma$ into $\Gamma)$. Thus, the affine map $(\{0,0,0\}, D): G \rightarrow G$ 
induces $\phi_{D}: \Gamma \backslash G \rightarrow \Gamma \backslash G$ and $\Phi_{D}: \Pi \backslash G \rightarrow \Pi \backslash G$ so that the following diagram is commutative:

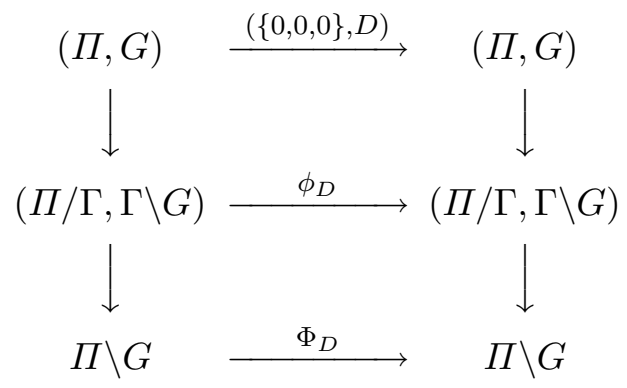

We take an ordered (linear) basis for the Lie algebra of $G$ as follows:

$$
\mathbf{e}_{1}=\left[\begin{array}{lll}
0 & 0 & 1 \\
0 & 0 & 0 \\
0 & 0 & 0
\end{array}\right], \quad \mathbf{e}_{2}=\left[\begin{array}{lll}
0 & 1 & 0 \\
0 & 0 & 0 \\
0 & 0 & 0
\end{array}\right], \quad \mathbf{e}_{3}=\left[\begin{array}{lll}
0 & 0 & 0 \\
0 & 0 & 1 \\
0 & 0 & 0
\end{array}\right]
$$

With respect to this basis, the differentials of $A$ and $D$ are

$$
A_{*}=\left[\begin{array}{ccc}
1 & 0 & 0 \\
0 & -1 & 0 \\
0 & 0 & -1
\end{array}\right], \quad D_{*}=\left[\begin{array}{ccc}
-1 & 0 & 0 \\
0 & 1 & 1 \\
0 & 1 & 0
\end{array}\right] \text {. }
$$

Therefore by Theorem 3.5, the Nielsen number of the map $\Phi_{D}: \Pi \backslash G \rightarrow$ $\Pi \backslash G$ is:

$$
\begin{aligned}
N\left(\Phi_{D}\right) & =\frac{1}{|\Psi|} \sum_{\psi \in \Psi} N\left(\psi \phi_{D}\right)=\frac{1}{|\Psi|} \sum_{\psi \in \Psi}\left|\operatorname{det}\left(I-\psi_{*} D_{*}\right)\right| \\
& =\frac{1}{2}\left(\left|\operatorname{det}\left(I-D_{*}\right)\right|+\left|\operatorname{det}\left(I-A_{*} D_{*}\right)\right|\right) \\
& =\frac{1}{2}(|-2|+|2|)=2 .
\end{aligned}
$$

Let $\alpha=(a, A)$. Since $(\{0,0,0\}, D) \alpha=\left(D(a) \cdot a^{-1}, I\right) \alpha(\{0,0,0\}, D)$ and $D(a) \cdot a^{-1} \in \Gamma$, we have $\phi_{D} \bar{\alpha}=\bar{\alpha} \phi_{D}$. This means that $\bar{\varphi}: \Pi / \Gamma \rightarrow \Pi / \Gamma$ maps $\bar{\alpha}$ to $\bar{\alpha}$. Hence $\bar{\varphi}$ is the identity map, and the Reidemeister classes of $\bar{\varphi}$ are singleton sets.

By Proposition 3.9,

$$
\begin{gathered}
\left|\operatorname{im}\left(\hat{i}_{\{0,0,0\}}\right)\right|=\frac{N\left(\phi_{D}\right)}{[\Pi: \Gamma]} \cdot|[\overline{1}]|=\frac{\left|\operatorname{det}\left(I-D_{*}\right)\right|}{2} \cdot 1=\frac{|-2|}{2} \cdot 1=1, \\
\left|\operatorname{im}\left(\hat{i}_{\alpha}\right)\right|=\frac{N\left(\bar{\alpha} \phi_{D}\right)}{[\Pi: \Gamma]} \cdot|[\bar{\alpha}]|=\frac{\left|\operatorname{det}\left(I-A_{*} D_{*}\right)\right|}{2} \cdot 1=\frac{|2|}{2} \cdot 1=1 .
\end{gathered}
$$


Every mod $\Gamma$ fixed point classes contains only one ordinary fixed point class. By Corollary 3.8,

$$
N_{\Gamma}\left(\Phi_{D}\right)=N\left(\Phi_{D}\right)=2 .
$$

ExAmPLE 3.11. Let $G$ be the 3-dimensional Heisenberg group. Let $K$ be the subgroup of $G$ consisting of all integral matrices of $G$. Let $a=$ $\left\{0,0, \frac{1}{4}\right\}$ and $A: G \rightarrow G$ be the automorphism of $G$ given by

$$
A(\{x, y, z\})=\{-x,-y, z\} .
$$

Then $A$ has period 2, and $(a, A)^{4}=\left(a^{4}, I\right)=(\{0,0,1\}, I) \in G \rtimes \operatorname{Aut}(G)$, where $I$ is the identity automorphism of $G$. The subgroup

$$
\Pi=\langle K,(a, A)\rangle \subset G \rtimes \operatorname{Aut}(G)
$$

generated by the lattice $K$ and the element $(a, A)$ is discrete and torsion free, and $K$ is a normal subgroup of $\Pi$ of index 4 . Note that $\Gamma=\Pi \cap G$ contains $(a, A)^{2}=\left(a^{2}, I\right)$ so that $K$ has index 2 in $\Gamma$.

Let $D \in \operatorname{Aut}(G)$ be given by

$$
D(\{x, y, z\})=\{3 y,-x, 3 z-3 x y\} .
$$

Then $(\{0,0,0\}, D)(a, A)(\{0,0,0\}, D)^{-1}=(a, A)^{3}$, and the conjugation by $(\{0,0,0\}, D)$ maps $\Pi$ into $\Pi$, and $K$ into $K$. Therefore, the affine map $(\{0,0,0\}, D): G \rightarrow G$ induces $\phi_{D}: K \backslash G \rightarrow K \backslash G$ and $\Phi_{D}: \Pi \backslash G \rightarrow \Pi \backslash G$.

With respect to the standard linear basis $\mathbf{e}_{1}, \mathbf{e}_{2}, \mathbf{e}_{3}$ for the Lie algebra of $G$, the differentials of $A$ and $D$ are

$$
A_{*}=\left[\begin{array}{ccc}
1 & 0 & 0 \\
0 & -1 & 0 \\
0 & 0 & -1
\end{array}\right], \quad D_{*}=\left[\begin{array}{ccc}
3 & 0 & 0 \\
0 & 0 & 3 \\
0 & -1 & 0
\end{array}\right] \text {. }
$$

Let $\alpha=(a, A) \in \Pi$. Then $\Pi / K$ is the cyclic group of order 4 generated by $\bar{\alpha}$. The Nielsen number of the map $\Phi_{D}: \Pi \backslash G \rightarrow \Pi \backslash G$ is, by Theorem 3.5:

$$
\begin{aligned}
N\left(\Phi_{D}\right)= & \frac{1}{[\Pi: K]} \sum_{\psi \in \Pi / K} N\left(\psi \phi_{D}\right) \\
= & \frac{1}{4}\left(N\left(\phi_{D}\right)+N\left(\bar{\alpha} \phi_{D}\right)+N\left(\bar{\alpha}^{2} \phi_{D}\right)+N\left(\bar{\alpha}^{3} \phi_{D}\right)\right) \\
= & \frac{1}{4}\left(\left|\operatorname{det}\left(I-D_{*}\right)\right|+\left|\operatorname{det}\left(I-A_{*} D_{*}\right)\right|\right. \\
& \left.\quad+\left|\operatorname{det}\left(I-D_{*}\right)\right|+\left|\operatorname{det}\left(I-A_{*} D_{*}\right)\right|\right) \\
= & \frac{1}{4}(|-8|+|-8|+|-8|+|-8|)=8 .
\end{aligned}
$$


Observe that the homomorphism $\bar{\varphi}: \Pi / K \rightarrow \Pi / K$ maps $\bar{\alpha}$ to $\bar{\alpha}^{-1}$. Its Reidemeister classes are $[\overline{1}]=\left[\bar{\alpha}^{2}\right]=\left\{1, \bar{\alpha}^{2}\right\}$ and $[\bar{\alpha}]=\left[\bar{\alpha}^{-1}\right]=\left\{\bar{\alpha}, \bar{\alpha}^{-1}\right\}$. By Proposition 3.9, $\left|\operatorname{im}\left(\hat{i}_{\alpha}\right)\right|=N\left(\bar{\alpha} \phi_{D}\right) /[\Pi: K] \cdot|[\bar{\alpha}]|=\frac{|-8|}{4} \cdot 2=4$. Every $\bmod K$ fixed point class consists of 4 ordinary fixed point classes. Hence

$$
N_{K}\left(\Phi_{D}\right)=2<8=N\left(\Phi_{D}\right) .
$$

Acknowledgement. The authors would like to thank the referee for pointing out some errors and making careful corrections of a few expressions in their original version.

\section{REFERENCES}

[1] D. V. Anosov, The Nielsen numbers of maps of nil-manifolds, Uspehi Mat. Nauk, 40 (1985), 133-134; Russian Math. Survey, 40 (1985), 149-150.

[2] B. Jiang, Lectures on Nielsen fixed point theory, Contemporary Mathematics 14, American Mathematical Society, Providence, R.I., 1983.

[3] J. B. Lee and K. B. Lee, Lefschetz numbers for continuous maps, and periods for expanding maps on infra-nilmanifolds, preprint (2003).

[4] K. B. Lee, Maps on infra-nilmanifolds, Pacific J. Math., 168 (1995), 157-166.

[5] C. K. McCord, Estimating Nielsen numbers on infrasolvmanifolds, Pacific J. Math., 154 (1992), 345-368.

[6] J. Shin, Isometry groups of unimodular simply connected 3-dimensional Lie groups, Geom. Dedicata, 65 (1997), 267-290.

Seung Won Kim

Department of Mathematics

Sogang University

Seoul 121-742

Korea

swkim@sogang.ac.kr

Jong Bum Lee

Department of Mathematics

Sogang University

Seoul 121-742

Korea

jlee@sogang.ac.kr

Kyung Bai Lee

Department of Mathematics

University of Oklahoma

Norman, OK 73019

U.S.A.

kb_lee@AFTERMATH.math.ou.edu 